\title{
Lien diasporique et création culturelle
}

\section{Un contexte favorable à la création culturelle}

L'usage du terme diaspora s'est considérablement élargi au cours des dernières décennies. Renvoyant initialement à un exil contraint, dans un cadre souvent tragique, il est aujourd'hui relativement banalisé et il suffit d'entretenir un lien, au moins symbolique avec un foyer originel et un ensemble de populations dispersées qui en sont issues pour se proclamer membre d'une diaspora. ${ }^{1}$

Le terme de black diaspora désignant les descendants des populations africaines ayant quitté le continent dans le cadre de la traite transatlantique pour être déportées dans diverses parties du continent américain, est apparu dans les années soixante, sous la plume de l'historien britannique George Shepperson ${ }^{2}$. II a fait l'objet de nombreux débats académiques dans le monde anglophone et n'a été importé que tardivement dans le monde francophone, ce qui ne signifie pas que l'objet qu'il désigne n'y était pas déjà présent. Nous n'allons pas entrer ici dans les polémiques autour de l'usage de ce terme pour désigner des populations originaires d'Afrique subsaharienne qui sont parties à diverses époques et dans des circonstances différentes pour arriver en Amérique puis en Europe. Nous nous limiterons à la question du lien entre le contexte diasporique et la production intellectuelle et artistique que suscite ce contexte.

Le fait de vivre en diaspora a des incidences sur la création culturelle. L'élargissement des horizons que procure l'expérience de la migration puis de l'installation, plus ou moins durable, en terre étrangère, favorisent l'expression du ressenti par des moyens littéraires, artistiques ou d'autres formes de langage aptes à cette expression. La rencontre de personnes qui partagent une même trajectoire depuis un même pays incite aux échanges et amène à trouver des formes d'expression individuelles ou collectives qui permettent d'exalter le souvenir de la terre des origines et à le faire partager par ceux qui y ont vécu ou par ceux, pour qui il n'est qu'une référence lointaine. Pour nourrir le lien diasporique, il faut entretenir la relation avec le pays commun sur un plan immatériel et symbolique à défaut de pouvoir le faire suffisamment par des aller retours et des échanges matériels. Cela est propre à inspirer des œuvres qui parlent des origines depuis les situations présentes, qui parlent du pays quitté depuis le pays d'arrivée et de toute l'expérience de la migration et des rencontres qu'elle a permises.

\section{Paris capitale de la diaspora noire}

La production culturelle suscitée par la conscience de vivre en diaspora a pris des contours différents, selon les époques et le profil des acteurs engagés dans cette production. On peut considérer que des années 1930 aux années 1950, Paris aura été la véritable capitale de la diaspora noire, autour d'un groupe d'intellectuels engagés pour faire reconnaître l'originalité culturelle du monde noir à travers leurs actions politiques mais surtout leurs œuvres littéraires. Ils ont un lieu de rencontre, le salon

- $1 \mathrm{~J}$ Barou; 2018 Les avatars du mot diaspora : du lien créé par le malheur commun à une catégorie de l'action publique Sens-Dessous 2018/1 (№ 21), pages 55 à 63

${ }^{2}$ G.Shepperson 1966 The African diaspora: or Africa abroad', African Forum, 2 
littéraire de Paulette Nardal et un organe d'expression, La revue du Monde noir. ${ }^{3}$ Ils n'utilisent jamais le mot diaspora mais leurs débats n'en contribuent pas moins à théoriser la relation immatérielle qui existe entre les différentes populations noires dispersées depuis le continent africain.

Ce public est en effet marqué par la diversité des origines. On y trouve les Sénégalais Léopold Sédar Senghor, à l'époque enseignant dans le secondaire et Birago Diop, étudiant vétérinaire, le Martiniquais Aimé Césaire, étudiant à l'Ecole Normale Supérieure, le Guyanais, Léon Gontran Damas, dandy anticonformiste, le Haïtien Jean Price Mars, médecin et ethnologue. Ces personnalités, toutes francophones, n'entretiennent pas moins des relations étroites avec des anglophones, écrivains, musiciens ou chanteurs. Le jazz commence à se répandre dans les caves de Saint Germain des Prés et Joséphine Baker triomphe avec le «bal nègre» de la rue Blomet. Cette diaspora noire qui s'ignore connaît alors une extraordinaire effervescence créative qui attirera plus tard de grands écrivains américains comme Richard Wright ou James Baldwin. Tous ces créateurs partagent un même souci d'affirmation de la part africaine de leur identité. Cela aboutira à la construction de la notion de négritude que Césaire définit ainsi : «La négritude est la simple reconnaissance du fait d'être noir, et l'acceptation de ce fait, de notre destin de Noir, de notre histoire et de notre culture» ${ }^{4}$

L'usage de ce concept dans un cadre politique et les critiques dont il a pu faire l'objet, de différents points de vue, ont tendance à occulter l'impact qui a été le sien sur le plan littéraire. Derrière la dénonciation de la domination vécue par leurs pays, on trouve chez ces auteurs engagés une quête des origines et une volonté d'inscrire les produits de leur culture parmi les grands courants créatifs de l'humanité. En publiant en 1947, les contes d'Amadou Koumba, Birago Diop ouvre la voie à un retour vers l'Afrique en faisant connaître à un large public des fragments de la littérature orale africaine, exprimés dans les langues locales. Senghor qui admirait énormément cet ouvrage écrira la préface d'une seconde édition en 1958. Parallèlement, ces auteurs fréquentent les bibliothèques et les musées, en particulier celui du Trocadéro, futur musée de l'Homme, en quête de connaissance sur un continent qui pour certains est celui de leur naissance et pour d'autres celui de la naissance de lointains ancêtres. En faisant lire à Césaire l'ouvrage du grand anthropologue allemand Léo Frobenius, Histoire de la civilisation africaine ${ }^{5}$, Senghor lui permet de découvrir sous une plume scientifique, une image de l'Afrique aux antipodes de la barbarie à laquelle elle était souvent réduite dans le discours des colonisateurs et aussi dans la perception des colonisés. Cela l'inspirera dans son discours sur le colonialisme pour dénoncer la négation de la valeur des cultures africaines. C'est donc bien l'Afrique qui constitue à l'époque l'infrastructure du lien diasporique au-delà de la référence à une identité noire, toujours associée à une identité de dominé et de discriminé. La découverte ou la redécouverte des civilisations africaines n'incite pas pour autant à un repli sur le passé. Comme l'écrit Pap Ndiaye :

\footnotetext{
${ }^{33}$ Femme de lettres née à la Martinique (1896/1985). Elle fonde avec sa sœur Jane un salon littéraire dans leur appartement de Clamart où elles reçoivent tout ce que Paris compte d'intellectuels et d'artistes originaires d'Afrique et des Antilles mais aussi des Etats Unis. En 1931, elle fonde La revue $d u$ monde noir, dont ne paraitront que huit numéros mais qui exprime déjà des débats d'idées que l'on retrouvera plus tard dans Présence Africaine, fondée en 1947 par Alioune Diop. Ces pionnières méconnues de la culture diasporique noire ont reçu en 2019 un début de reconnaissance de la part de la ville de Paris qui a donné leur nom à une promenade du $14^{\mathrm{e}}$ arrondissement reliant la petite ceinture à la coulée verte Vercingétorix.

${ }^{4}$ A Césaire, 1947, Cahier d'un retour au pays natal, Bordas.

${ }^{5}$ L.Frobenius, 1936 Histoire de la civilisation africaine, Gallimard.
} 
"il ne s'agissait pas tant de revenir aux civilisations anciennes que d'inscrire l'africanité culturelle dans la modernité et dans un humanisme universaliste. ${ }^{6}$

\section{Premières fragmentations}

Il n'y a en effet aucune tentation sectaire dans ces affirmations identitaires diasporiques. Ouverts sur l'ensemble du monde noir, les auteurs de cette époque sont aussi ouverts sur l'ensemble du monde et de nombreux intellectuels français parmi les plus prestigieux de l'époque, de Sartre à Camus, en passant par Gide appuient leur démarche pour affirmer la valeur de leurs cultures et leurs aspirations à la liberté. Mais au fil des années 1950, la diaspora noire commence à se fracturer. Avec les indépendances de la plupart des pays africains, les intellectuels militants du quartier latin voient se briser leurs rêves de panafricanisme. Si tout le monde condamne la balkanisation de l'Afrique, personne ne parvient à constituer des fédérations viables, les rêves d'unité se heurtant à la réalité des ambitions personnelles. Ceux qui, comme Senghor, avaient déjà eu des mandats sous l'Union Française, se retrouvent à devoir construire des états nations dans un cadre où l'ancienne puissance tutélaire garde une influence encore décisive. Du côté des Antillais, le choix de Césaire d'opter pour la départementalisation, conduit à une intégration renforcée à la République et à une réduction des liens avec les divers états de la Caraïbe anglophone. La diaspora noire perd de son ampleur tandis que la migration des Antillais vers la métropole renforce le processus d'assimilation. Aux Etats Unis, la production littéraire et artistique des Africains américains se concentre sur les souffrances et les injustices qui marquent la vie quotidienne d'une partie de la population. Leur combat se partage entre la lutte pour les droits civiques qui relève d'une logique de quête d'intégration et de revendication d'égalité et une affirmation identitaire qui s'appuie sur le seul fait d'être noir, sans références à une culture particulière ni à une terre d'origine précise. Cette deuxième tendance a pu déboucher parfois sur un nationalisme sectaire. La couleur est comprise non pas comme un héritage biologique mais comme une construction sociale effectuée par les groupes dominants qui vise à la marginalisation de cette population. C'est là que se creuse une différence entre ce que l'on peut appeler la diaspora africaine et la diaspora noire. Dans le premier cas, l'accent est mis sur la référence à l'Afrique, continent en souffrance mais riche de potentialités d'avenir. Dans le second cas, l'accent est mis sur l'expérience d'une discrimination par la couleur qui se renouvelle sous diverses formes depuis la réduction en esclavage jusqu'à nos jours. L'Afrique n'est pas absente de la créativité littéraire des intellectuels noirs d'Amérique et des Antilles mais elle est facilement mythifiée et idéalisée à partir d'une très faible expérience de sa réalité. En 2007, la parution d'un roman intitulé : Lose Your Mother ; a Journey along the Atlantic Slave Coast ${ }^{7}$ révèle un sentiment de quasi impossibilité de se reconnecter avec le continent des origines. L'auteure, Saidiya Hartman, universitaire newyorkaise d'origine antillaise, ayant une formation en sciences humaines a mené un véritable travail ethnographique et historique sur les routes empruntées par les esclaves raflés à l'intérieur des terres et les forts où ils ont été détenus dans l'attente de leur transfert vers l'Amérique. Elle a cherché tous les témoignages possibles sur les origines de cette tragédie. A l'issue de ce travail, elle constate une quasi impossibilité de retour vers la terre ancestrale, terre fantasmée comme lieu de ressourcement affectif mais qui abrite des Africains qui ont vendu d'autres Africains et qui aujourd'hui cherchent à profiter sans vergogne des touristes en quête de leurs origines qu'ils regardent comme des nantis qu'il faut exploiter en leur vendant de pseudo souvenirs.

\footnotetext{
${ }^{6} \mathrm{P} ; \mathrm{Ndiaye}, 2009$, « Présence africaine avant «Présence Africaine» : la subjectivation politique noire en France dans l'entre-deux-guerres », Gradhiva, n 10, p 78

${ }^{7}$ S. Hartman, 2008, Lose Your Mother ; a Journey along the Atlantic Slave Coast, Farrar Straus and Giroux eds, Nez York; 270 pages
} 


\section{Le regard de l'Amérique}

De telles conclusions sont rares dans la littérature diasporique qui tend toujours à entretenir et à magnifier le lien avec l'Afrique, bien que beaucoup aient conscience de son caractère fictif, parce que sans lui, la diaspora perd de son contenu. Selon le contexte politique, l'engouement des intellectuels de la diaspora noire pour le continent africain connaît des hauts et des bas. Au cours des dernières années, plusieurs auteurs américains se sont installés au Ghana, suivant ainsi un mouvement plus large au sein de la population africaine américaine et encouragé par les autorités ghanéennes qui y voient surtout un intérêt économique. Le choix de ce pays n'est pas neutre. Le Ghana a été l'un des principaux pays de départ des navires négriers vers les colonies anglaises d'Amérique du Nord et il est probable que beaucoup d'Africains américains y aient leurs origines. Mais au-delà de ce pèlerinage rituel sur les traces des ancêtres qu'il peut offrir, le Ghana apparaît comme cumulant de nombreux symboles positifs. Premier pays d'Afrique subsaharienne à devenir indépendant en 1957, sous la présidence de Kwamé N'Krumah, chantre du panafricanisme, il est aussi une des rares nations du continent où la démocratie semble s'être durablement implantée. La visite officielle qu'y a faite Barrack Obama en 2009 a placé dans la lumière ce pays qui possède un potentiel touristique important. En 2019, la célébration de « l'année du retour », commémorant les 400 ans des premiers débarquements d'esclaves sur les côtes de Virginie a été astucieusement mise en scène et a attiré nombre de personnalités afro descendantes venues des Antilles, des Etats Unis, de Grande Bretagne et même de France ${ }^{8}$. L'engouement pour une terre incarnant à la fois le passé et l'avenir de la diaspora noire survivra-t-il à l'évènement et Accra jouera-t-elle au XXIe siècle, le rôle de Paris, cent ans auparavant comme lieu de création et d'échanges intellectuels et artistiques exaltant le lien diasporique ? Il n'est pas interdit de tenter de l'imaginer.

\section{Itinérances africaines aux Etats Unis}

Pour évaluer les chances que se reconstitue ce lien, il est intéressant de suivre la trajectoire de certains intellectuels africains qui font de plus en plus souvent le chemin vers leurs frères de la diaspora qui résident aux Etats-Unis. Leur regard révèle quelques failles dans le tissu diasporique et la conscience d'une distance difficile à réduire entre eux et les Africains américains. Dans son roman Américanah", l'écrivaine nigériane, Chimamanda Ngozi Adichie met en scène une jeune femme qui part étudier aux Etats-Unis, sans le confort d'une bourse. Elle y vit la vie de débrouille caractéristique de la précarité des migrants pauvres et de nombreuses aventures amoureuses avec des amants aussi bien blancs que noirs. C'est surtout le comportement de ces derniers qui l'interroge. Elle rapporte une discussion entre étudiants, menée par un Kenyan, à propos des relations entre Africains et Africains Américains :

"Notez qu'en général, les Afro-Américains adhèrent au syndicat des étudiants noirs et les Africains à l'Association des étudiants africains...Les Afro-américains qui viennent à nos réunions écrivent des poèmes à notre mère l'Afrique et pensent que chaque Africaine est une reine de Nubie. Si un Afro américain vous appelle Mandingo ou vous traite de peau de fesses, il vous insulte parce que vous êtes africain...Vous découvrirez que vous pouvez vous lier plus facilement avec d'autres expatriés, Coréens, Indiens, Brésiliens, peu importe, qu'avec des Américains, noirs ou blancs. ${ }^{10}$

Pour exprimer ses interrogations sur ces relations quelque peu inattendues, l'hérö̈ne décide de créer un blog dans lequel elle s'adresse à ses camarades noirs non américains qui sont, comme elle, surpris

\footnotetext{
${ }^{8}$ L.Filippi, 2019, Au Ghana, les Afro Américains retournent sur les traces des esclaves déportés outre Atlantique, www.francetvinfo.fr, 2 sept 2019.

${ }_{9}^{9}$ C.Ngozie Adichie, 2013, Américanah, Gallimard, collection folio, traduit de l'anglais par Anne Damour $p$

${ }^{10}$ Idem p 213.
} 
de se voir systématiquement accolé une identité de noir alors que, dans leur pays d'origine, ils ne se définissaient jamais ainsi :

"Cher noir non américain, quand tu viens en Amérique, tu deviens noir. Cesse de discuter. Cesse de dire je suis jamaïcain ou je suis ghanéen; L'Amérique s'en fiche. Quelle importance si tu n'es pas noir chez toi.»"${ }^{11}$

Son regard extérieur révèle le poids de cette identité restrictive souvent intériorisée par ceux à qui elle s'est imposée. Cet ouvrage de fiction interroge la question du lien diasporique et amène à douter de sa possibilité mais en même temps, c'est grâce au contexte de diaspora, qu'un tel écrit est possible.

Dans son carnet de bord Rumeurs d'Amérique ${ }^{12}$, Alain Mabanckou, qui vit aux Etats-Unis depuis le début des années 2000, témoigne aussi de son étonnement devant le poids de cette identité noire qui conduit à s'imposer des formes d'apartheid, mais il se montre plus optimiste en constatant que les dérives racistes de la présidence Trump ont fini par provoquer un mouvement de solidarité inédite de toute la population après l'accumulation de bavures visant les noirs et l'absence ostensible de compassion de la part des autorités. Au-delà ce qui rapproche les membres éparpillés d'une diaspora, il y a toujours, à un moment un élargissement de la solidarité à l'ensemble de la population. Mais c'est aussi dans un contexte de diaspora que cela peut se ressentir le plus fortement.

\section{Représentations populaires de la diaspora}

Le sentiment de diaspora n'est pas exprimé par les seuls intellectuels qui usent des moyens de production culturelle quelque peu élitistes que sont les arts et la littérature. Dans son ouvrage The Black Atlantic, modernity and double consciousness ${ }^{13}$, le sociologue britannique Paul Gilroy a défini la diaspora noire comme hybride, faite de mélanges entre des éléments africains, américains, caraïbes et britanniques. Les moyens de circulation contemporains ont permis à un certain nombre de gens de cultiver cette double appartenance à la fois au pays où ils résident et à cet univers plus large de la diaspora qui s'appuie moins sur des fondements situés en Afrique que sur la référence à l'Atlantique comme séparation des deux univers mais aussi comme moyen de les relier. Cette double conscience est surtout exprimée par des intellectuels engagés dans le projet lointain d'unification des populations noires dispersées, gardant leur originalité, mais affichant une référence commune à l'histoire de leur dispersion et une volonté de compter dans le concert des nations.

Christine Chivalon, spécialiste de la Caraïbe anglophone observe que le discours des intellectuels engagés n'est peut-être pas la seule vision existante de la diaspora noire et qu'il serait bon $d^{\prime}$ 'interroger les représentations que peuvent en avoir des milieux plus populaires.

"On peut se demander si le choix de Gilroy consistant à privilégier le discours de quelques figures de la sphère politique et littéraire, était le bon. Que dire d'une sphère plus " ordinaire ", celle où se construit le "culturel " au quotidien ? N'est-ce pas là que se définissent les termes d'une appartenance sociale dont le discours politique et littéraire n'est qu'une des modalités de mise en représentation? "14

\footnotetext{
${ }^{11}$ Idem p 330

${ }^{12}$ A. Mabanckou, 2020, Rumeurs d'Amérique, Plon.

${ }^{13}$ P, Gilroy, 1993; The Black Atlantic, modernity and double consciousness, Harvard University Press

${ }^{14}$ Christine Chivallon, 2002. La diaspora noire des Amériques, réflexion sur le modèle d'hybridité de Paul Gilroy, L'Homme, $n^{\circ} 161, p 60$.
} 
Le culturel qui se construit au quotidien et qui relève d'une créativité collective est le fait des milieux populaires qui ont de la diaspora une représentation plus limitée mais plus concrète. Leur référence principale est avant tout une diaspora ethnique qui a conservé, par-delà les mers des liens qu'il est encore possible d'activer. Cela explique la quasi absence des auteurs noirs hispanophones et lusophones dans les débats sur l'identité noire diasporique, depuis les années de l'effervescence parisienne jusqu'aux discussions autour de l'ouvrage de Gilroy. Vivant dans des sociétés beaucoup plus métissées, ils ont aussi sous les yeux l'exemple d'un sentiment diasporique vécu par les milieux populaires qui est encore très vivant à travers les anciennes religions africaines pratiquées de part et d'autre de l'Atlantique sud.

\section{Diasporas ethniques}

Les rituels, religieux ou profanes, la littérature orale, les expressions du corps dans la danse et la transe comptent parmi les principaux moyens d'activer le lien diasporique. Les travaux d'Herskovits ${ }^{15}$ et de Bastide $^{16}$ ont montré que ceux qui entretiennent ce type de lien se trouvent surtout au Brésil et dans la Caraïbe hispanophone. Les Africains transférés dans ces pays étaient pour beaucoup d'ethnie yoruba et ont amené avec eux une religion bien structurée avec une hiérarchie cléricale très élaborée, des rites initiatiques complexes et un panthéon comptant d'innombrables Orixa, demi dieux incarnant les diverses forces de la nature à propos desquels on n'en finit plus d'imaginer des récits mythiques et légendaires. Le fait de se retrouver en milieu catholique a permis le maintien du culte des Orixa. Pour les missionnaires désireux de convertir les esclaves, il était facile de puiser parmi les nombreux saints du panthéon catholique pour y trouver des figures correspondant à tel ou tel Orixa. Culte des saints et culte des génies sont allés de pair dans des créations syncrétiques infiniment renouvelées. Quels qu'aient pu être les apports chrétiens, le fond religieux africain est demeuré et a permis d'entretenir le rapport symbolique à la terre des origines par l'élaboration de nouveaux mythes comme celui de l'arbre qui traverse l'océan pour joindre les deux mondes et le long duquel les Orixa circulent quand on les appelle pour venir «chevaucher» leurs adeptes pendant les séances de possession. C'est aussi par le biais de cet arbre transocéanique que l'âme des morts retourne sur le continent africain tandis que leurs corps sont enterrés près des Candomblés, « " petits morceaux d'Afrique plantés en plein cœur du Brésil. Non de l'Afrique profane mais de l'Afrique mystique ". ${ }^{17}$

Culture et religion se confondent dans cette créativité de l'imaginaire collectif. A La Havane le musée des Orixa construit avec l'aide de l'Etat cubain en plein centre-ville est aussi le siège de l'association culturelle yoruba, reconnue par les pouvoirs publics comme composante de la culture cubaine. Ce musée n'est pas seulement un lieu où s'exposent de nombreuses sculptures et peintures représentant les plus connus des Orixa, mais il sert aussi de temple pour les cérémonies de santéria, ce culte syncrétique où saints du catholicisme et génies africains se confondent.

\section{Inventivité collective et ouverture}

C'est aussi par le biais de la dynamique de création culturelle, orchestrée par des associations dirigées par des personnalités reconnues, que les immigrés africains en France et une part de leurs descendants parviennent à faire vivre le lien diasporique. C'est ainsi que les Soninké, venus travailler en France pendant les années 1960, ont au fil du temps œuvré pour le maintien de l'usage de leur langue

\footnotetext{
${ }^{15}$ M.J Herskovits 1966 L'héritage du Noir : mythe et réalité, Présence africaine.

${ }^{16}$ R. Bastide, 1960, Les religions africaines au Brésil, vers une sociologie des interpénétrations des civilisations. PUF.

${ }^{17}$ R.Bastide, 2001, Le Candomblé de Bahia, transe et possession du rite du Cadomblé, p 91, Terre Humaine, Poche.
} 
et de leur culture et ont tissé des liens avec les communautés éparpillées dans divers pays d'Afrique. Dans le cas de cette population, l'idée de diaspora est ancienne. Les mythes fondateurs parlent de la dispersion provoquée par l'effondrement du royaume de Wagadou, correspondant probablement à l'empire de Ghana (IIIe/XIIIe siècle) situé entre l'actuelle Mauritanie et le Mali. L'ancienneté de la dispersion est revendiquée aujourd'hui pour asseoir l'identité d'un peuple migrant qui a semé des communautés dans toute l'Afrique de l'ouest avant de venir en Europe. Les créations culturelles collectives s'adaptent à la modernité, passant par l'organisation de festivals dans les capitales des pays d'origine de ce peuple qui mettent en scène des spectacles vivants inspirés de son histoire et de son présent. (voir dans ce numéro, l'entretien donné par Ousmane Diagana, président de l'Association pour la Promotion du Soninké). Pas plus que dans le cas des Diola (voir p 189 l'article de Mariame Bodian), il n'y a chez les Soninké une tentation de fermeture sur une identité ethnique exclusive. Ces groupes absorbent de nombreux emprunts à la culture de leur milieu d'accueil et ne craignent pas d'intégrer des étrangers à leurs manifestations. Leur horizon est certes plus limité que celui des intellectuels qui rêvent d'un panafricanisme, rassemblant toute la diaspora noire de la planète, mais leur engagement débouche sur des actions concrètes qui maintiennent la vitalité du lien diasporique et expérimentent des formes d'expression culturelle bien adaptées au contexte d'un monde où les mobilités sont soutenues et touchent autant les milieux populaires que les élites. Ainsi se maintient une conscience diasporique propice à la création culturelle et à l'ouverture au monde.

Jacques Barou

\section{CNRS}

